\section{Creencias de hombres de Cali, Colombia, sobre el examen digital rectal: hallazgos de un estudio exploratorio}

\author{
Beliefs of men from Cali, Colombia, \\ about digital rectal examination: \\ findings of an exploratory study
}

Diego Iván Lucumí-Cuesta 1

Gustavo Alonso Cabrera-Arana ${ }^{2}$

\footnotetext{
1 Fundación FES Social, División de Salud, Bogotá, Colombia.

2 Facultad Nacional de Salud Pública, Universidad de Antioquia, Medellín, Colombia.

Correspondencia D. I. Lucumí-Cuesta Fundación FES Social, División de Salud. Carrera 7 , No. 73-55, Oficina 1202, Bogotá, Colombia. dilucumi@fundacionfes.org
}

\begin{abstract}
This study used the Health Beliefs Model to explore beliefs on digital rectal examination (DRE) to screen for prostrate cancer, prior to intent to submit to the examination, in men 45 to 64 years of age in Cali, Colombia. The 267 participants answered a questionnaire by means of prior selection through proportional sampling by quotas. A univariate and bivariate analysis showed that only $25.8 \%$ of the participants had submitted previously to digital rectal examination and that $68.1 \%$ intended to do so in the following 12 months. Significant differences $(p<$ 0.05 ) were found between a history of DRE and schooling, socioeconomic status, health system coverage, perception of susceptibility to the disease, and perception of barriers and benefits. Significant differences $(p<0.05)$ were found between intent to submit to DRE and health system coverage, perception of severity of the disease, and perception of barriers. Given the low level of history of DRE and intent to submit to the examination, the results could help improve strategies to increase this practice at the local level.
\end{abstract}

Neoplasm; Prostate; Men's Health

\section{Introducción}

En Colombia, como en otros países del mundo, el perfil de morbimortalidad por cáncer en la próstata ha cambiado en las últimas décadas $1,2,3,4,5$.

Entre los casos de neoplasia maligna identificados en hombres mayores de 65 años en el principal centro de diagnóstico y atención a pacientes con cáncer del país, después de la piel, las localizaciones más frecuentes de cáncer son la próstata y el estómago 1,6. Según el Registro Poblacional de Cáncer de Cali, único de su clase en Colombia, del total de casos diagnosticados en hombres de todas las edades, el localizado en la próstata pasó de representar un 8,9\% en el quinquenio $1962 / 1967$ a un $20,1 \%$ en el quinquenio 1992/1996, ocupando ahora el primer lugar entre casos de cáncer diagnosticados en hombres de la ciudad, con una tasa de 27,5 casos por 100 mil habitantes ?.

$\mathrm{Al}$ igual que para otras localizaciones de cáncer, los factores de riesgo que han mostrado una mayor asociación con el de la próstata son inmodificables (edad, raza, antecedente familiar), razón que ha limitado el desarrollo de medidas efectivas de prevención primaria, haciendo de las de prevención secundaria la alternativa a ofrecer a la población en riesgo 8,9.

El examen digital rectal (EDR) y el antígeno prostático específico (PSA, por su nombre en inglés), han sido los dos principales métodos 
empleados en la prevención secundaria, con la tamización periódica a hombres mayores de 50 años con una expectativa de vida mayor de 10 años, previa discusión con el médico de los riesgos y beneficios de su práctica. A pesar de la amplia controversia que ha habido frente a la tamización, su práctica se ha sustentando en la recomendación de diferentes organizaciones de carácter mundial 9 .

En Colombia, la reglamentación vigente del Sistema General de Seguridad Social en Salud (SGSSS) establece que para los hombres afiliados mayores de 45 años la realización del EDR debe ser al menos cada cinco años 10,11. Sin embargo, en el sistema existe una limitada información sobre la prevalencia de realización de esta práctica, al igual que sobre los factores que la condicionan.

Los factores que condicionan la adopción de comportamientos saludables y preventivos, como la tamización, pueden ser múltiples, dado que esta no sólo depende de su oferta o inducción por parte de los organismos de salud del SGSSS. Las personas pueden tomar decisiones relacionadas con prácticas preventivas según la percepción que tienen y las valoraciones hechas en forma individual o grupal sobre las consecuencias que se derivan de tales prácticas 12 , por tanto, estas percepciones deberán ser consideradas para el diseño de programas de tamización efectivos 13 .

Teniendo en cuenta que la reglamentación colombiana solamente cobija para tamización del cáncer en la próstata la realización del EDR 10,11, se desarrolló un estudio en dos componentes, uno cualitativo y otro cuantitativo, con el fin de explorar las creencias que sobre dicha práctica tenían hombres entre de 45 a 64 años de Cali, Colombia, y caracterizar sus antecedentes de realización e intención de práctica durante los siguientes 12 meses. Se usó como referente teórico el Modelo de Creencias en Salud (MCS) (Figura 1). El MCS se fundamenta en la valoración subjetiva de una determinada expectativa, en la cual el valor es el deseo de evitar el padecimiento o enfermedad y, la expectativa en que una acción posible de realizar prevendrá o mejorará el proceso 14 . El modelo original se propuso según cuatro constructos: susceptibilidad, severidad, beneficios y barreras percibidas, que fueron complementados posteriormente por los conceptos claves para actuar, factores modificantes y auto-eficacia 12 y ha sido ampliamente usado para conducir procesos de intervención e investigación en salud pública, especialmente relacionados con pruebas de tamización 14,15.

\section{Métodos}

\section{$\underline{\text { Lugar de estudio }}$}

El estudio se llevó a cabo en Santiago de Cali, que cuenta con aproximadamente 2,2 millones de habitantes 16. La ciudad está ubicada al suroccidente del país y es la capital del departamento del Valle del Cauca, uno de los 32 entes territoriales en que se divide Colombia.

El estudio comprendía un componente cualitativo y otro cuantitativo, los cuales se llevaron a cabo a lo largo del año 2002. Los resultados del componente cualitativo, primero en ejecutarse, fueron publicados previamente 17 , por tanto, en este artículo se describen solamente los resultados del componente cuantitativo. Ambos componentes fueron ejecutados previo aval de los comités de ética de la Facultad de Salud de la Universidad del Valle y el Programa de Subvenciones a la Investigación de la Organización Panamericana de la Salud (OPS).

\section{Tamaño de muestra y selección de participantes}

Por la naturaleza exploratoria del estudio se optó por un muestreo de cuotas proporcional, teniendo como referencia los seis estratos socioeconómicos en los que se divide la ciudad de acuerdo al sistema de estratificación socioeconómica de Colombia. Sólo con el fin de establecer un número total de participantes suficiente para cada cuota y sin el propósito de hacer inferencias, se usó un tamaño de muestra de 266, el cual había sido uno de los previamente calculados ante la posibilidad de realizar un estudio de tipo probabilístico.

La captación de los participantes se hizo por medio de encuestadoras con formación en el área social debidamente capacitadas. Previa identificación de lugares de alta concurrencia (parques, centros comerciales, iglesias, etc.) por hombres en barrios seleccionados por conveniencia y representativos de cada estrato, las encuestadores invitaban a participar individualmente a los hombres, a quienes se les presentaba el objetivo del estudio, se verificaba que tuvieran entre 45 a 64 años de edad, habitaran en una residencia urbana del respectivo estrato y no presentaran ninguna condición física o mental que impidiera su participación; en quienes aceptaban participar se obtenía su consentimiento y se diligenciaba el cuestionario. Al finalizar la encuesta se entregaba a cada participante un volante con información sobre la enfermedad, que estimulaba asistir al servicio de salud para discutir con el médico lo relacionado con el EDR. 


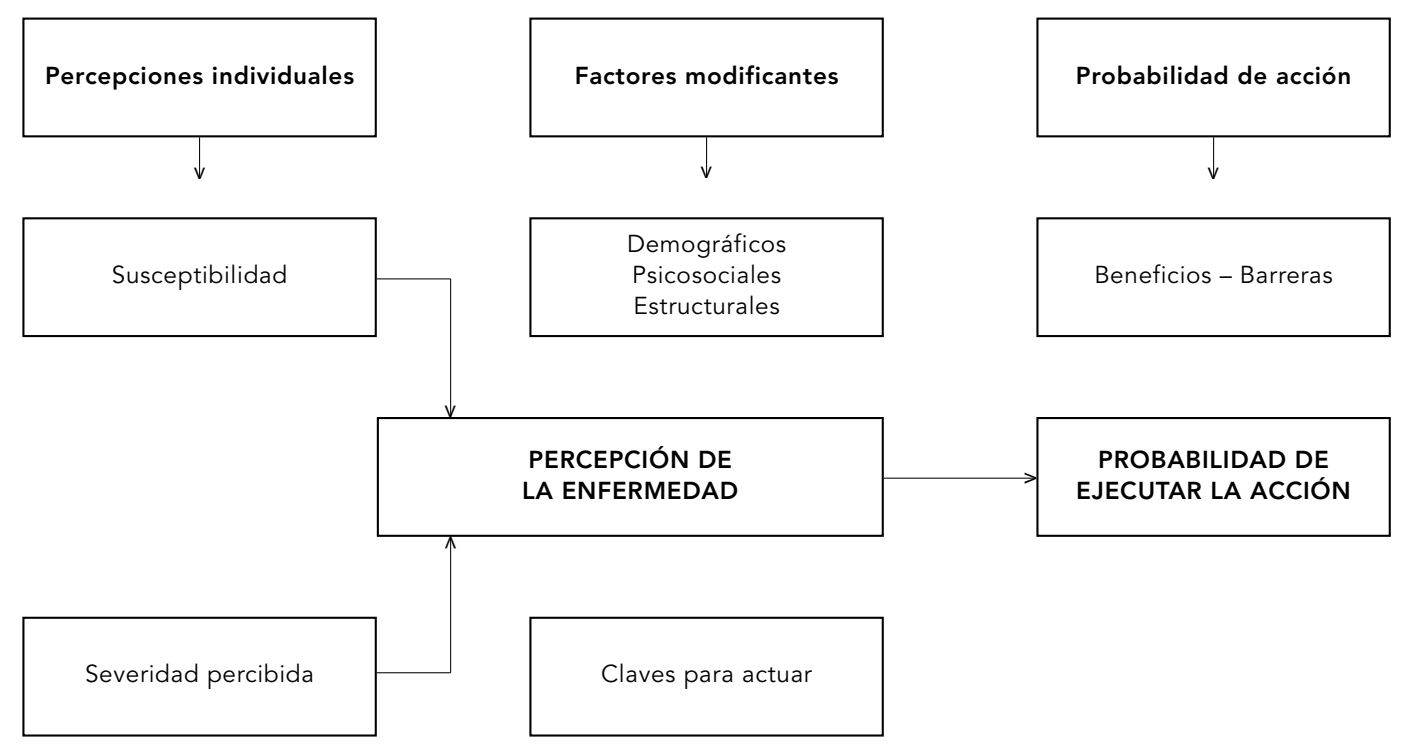

Fuente: Strecher \& Rosentock 12.

El investigador principal revisó cada cuestionario para verificar su diligenciamiento correcto. Para confirmar la veracidad de los datos se seleccionó al azar al $10,0 \%$ de los participantes con el fin de hacerles una reentrevista telefónica.

\section{Diseño del cuestionario}

Por la ausencia de cuestionarios validados en Colombia sobre el tema, se construyó uno inicial teniendo como referencia los hallazgos del componente cualitativo 17. Mediante la técnica Delphi se convocó a cuatro profesionales, expertos nacionales en la conducción de programas de salud sexual y reproductiva, prevención de enfermedades crónicas y educación en salud, y después de tres rondas se llegó a un acuerdo sobre la versión del cuestionario que fue sometida a una prueba piloto para su ajuste final. El instrumento definitivo tuvo 28 preguntas agrupadas en cuatro bloques; los tres primeros correspondientes a las variables independientes y el último a las dos variables dependientes.

En el primer bloque se indagó sobre variables sociodemográficas: edad, nivel de escolaridad, estrato socioeconómico, estado de afiliación al SGSSS. Para efectos del análisis la edad fue dividida en dos grupos: 45 a 54 y 55 a
64; para la escolaridad por su parte se tuvieron en cuenta los tres principales rangos en los cuales se pueden agrupar los grados de escolaridad cursados por una persona en Colombia. Para el estrato socioeconómico se establecieron tres grupos: bajo (estratos I y II) medio (estratos III y IV) y alto (estratos V y VI). Finalmente, quienes dijeron pertenecer alguno de los regímenes de salud establecidos en el SGSSS fueron considerados como afiliados, mientras que quienes informaron no pertenecer a ninguno fueron considerados como no afiliados.

El segundo bloque del cuestionario contenía preguntas relacionadas con el tipo y forma de obtención de información sobre el cáncer de la próstata y el EDR. El tercero, contenía las variables iniciales del MCS, las cuales fueron definidas con fines operativos teniendo a partir de sus definiciones conceptuales: (1) susceptibilidad percibida: probabilidad reconocida de presentar cáncer de próstata; (2) severidad percibida: gravedad que el individuo asigna a la enfermedad, su tratamiento y no tratamiento; (3) barreras percibidas: aspecto percibido como impedimento para someterse al EDR y; (4) beneficios percibidos: ganancias resultantes de la práctica del EDR 12.

Las variables dependientes fueron el antecedente y la intención de práctica, indagadas 
con las siguientes preguntas: “ ¿Hace cuánto se sometió al examen digital rectal para determinar el estado de su próstata?” y “¿En los próximos 12 meses tiene usted la intención de someterse al examen digital rectal?".

\section{Análisis estadístico}

El programa estadístico Stata fue utilizado para realizar dos tipos de análisis. El primero, descriptivo, sobre características sociodemográficas; y el segundo, bivariado, examinando la independencia entre las variables independientes y las dependientes usando tablas $2 \mathrm{xN}$ y el test de chi cuadrado o de Fisher cuando fue necesario.

\section{Resultados}

Se diligenciaron 267 cuestionarios, siete de los cuales fueron anulados una vez se realizó el control de calidad previo a la digitación de los datos, sin que esto afectara las proporciones previstas para cada cuota. Tal como se presenta en la Tabla 1, la mayoría de los participantes eran menores de 55 años $(61,5 \%)$, carecían de educación secundaria o superior $(62,0 \%)$, perte-

Tabla 1

Características sociodemográficas de hombres
de 45-64 años participantes en el estudio.
Cali, Colombia, 2002 .

\begin{tabular}{lcc}
\hline Características & Frecuencia & $\%$ \\
\hline Edad (años) & & \\
$45-54$ & 100 & 31,5 \\
$55-64$ & & \\
& & \\
Nivel de escolaridad & 161 & 62,0 \\
$\quad$ Primaria o menos & 62 & 24,0 \\
Secundaria & 37 & 14,0 \\
$\quad$ Técnico y superior & & \\
& & \\
Estrato socioeconómico & 159 & 61,0 \\
Bajo (1 y 2) & 69 & 27,0 \\
Medio (3 y 4) & 32 & 12,0 \\
Alto (5 y 6) & & \\
Afiliación al SGSSS* & & 34,7 \\
Sí & 90 & \\
No & &
\end{tabular}

necían al estrato socioeconómico bajo (61,0\%) y estaban afiliados al SGSSS (65,3\%).

En la Tabla 2 se observa que en cuanto al antecedente de práctica del EDR hubo diferencias estadísticamente significativas en el nivel de escolaridad, el estrato socioeconómico, estado de afiliación al SGSSS, la susceptibilidad y barreras percibidas. Mientras para la intención de práctica en los próximos 12 meses, se encontraron diferencias estadísticamente significativas en el estado de afiliación al SGSSS, severidad, beneficios y barreras percibidas.

Al explorar sobre barreras para la realización del EDR, 210 participantes $(81,0 \%)$ percibieron alguna, principalmente de tres tipos: bajo autocuidado $(29,0 \%)$, desarrollo asintomático de la enfermedad $(14,0 \%)$ e incomodidad del examen (14,0\%). De otro lado, los principales beneficios identificados por 253 de los participantes $(98,0 \%)$ fueron la tranquilidad de saber que no tienen la enfermedad $(46,0 \%)$, seguido por considerar que el EDR previene el cáncer (30,0\%).

Con respecto a la información, 51,0\% de los hombres dijo no haber recibido información con respecto al cáncer en la próstata y 53,0\% no había recibido información relacionada con el EDR; 40,0\% dijo no tener información sobre ninguno de los dos aspectos. Para los 159 hombres $(60,0 \%)$ que dijeron haber recibido información sobre la enfermedad, el EDR o ambos, el personal de salud y los medios de comunicación fueron las principales fuentes.

\section{Discusión}

Este es uno de los primeros estudios que se reporta en Latinoamérica sobre el antecedente e intención de práctica del EDR y sus resultados pueden, tomando las precauciones debidas, contribuir a orientar estrategias locales de prevención del cáncer en la próstata.

Sólo una cuarta parte de los participantes manifestaron un antecedente de práctica del EDR y, aunque la intención de someterse a su realización en los próximos 12 meses fue en relación al antecedente mayor, esta también puede considerarse como baja, pues los participantes estaban en una edad en la cual la práctica de esta prueba preventiva es recomendada y no tiene costo para los afiliados al SGSSS. No obstante, las diferencias socioculturales y en la estructura de prestación de los servicios preventivos en salud, los hallazgos encontrados en este estudio con respecto al porcentaje de hombres que se han realizado o tienen la intención de realizarse el EDR, son similares o inferiores 
Proporción de hombres con antecedente e intención de práctica del examen digital rectal en los próximos 12 meses, según características sociodemográficas y constructos del Modelo de Creencias en Salud. Cali, Colombia, 2002.

\begin{tabular}{|c|c|c|c|c|c|c|}
\hline \multirow[t]{2}{*}{ Características } & \multicolumn{3}{|c|}{ Antecedente de práctica (\%) } & \multicolumn{3}{|c|}{ Intención de práctica (\%) } \\
\hline & Sí & No & $p$ & Sí & No & $p$ \\
\hline Total de participantes & 25,8 & 74,2 & - & 68,1 & 31,9 & - \\
\hline \multicolumn{7}{|l|}{ Edad (años) } \\
\hline $45-54$ & 24,3 & 75,7 & 0,51 & 63,7 & 36,3 & 0,07 \\
\hline $55-64$ & 28,0 & 72,0 & & 75,0 & 25,0 & \\
\hline \multicolumn{7}{|l|}{ Nivel de escolaridad } \\
\hline Primaria o menos & 17,4 & 82,6 & 0,00 & 67,1 & 32,9 & 0,78 \\
\hline Secundaria & 30,7 & 69,3 & & 67,8 & 32,2 & \\
\hline Técnico y superior & 54,1 & 45,9 & & 73,0 & 27,0 & \\
\hline \multicolumn{7}{|l|}{ Estrato socioeconómico } \\
\hline Bajo (1 y 2) & 15,0 & 85,0 & 0,00 & 62,9 & 37,1 & 0,07 \\
\hline Medio (3 y 4 ) & 33,3 & 66,7 & & 75,4 & 24,6 & \\
\hline Alto $(5$ y 6$)$ & 62,5 & 37,5 & & 78,2 & 21,8 & \\
\hline \multicolumn{7}{|l|}{ Afiliación al SGSSS* } \\
\hline Sí & 31,0 & 69,0 & 0,01 & 72,8 & 27,2 & 0,02 \\
\hline No & 17,0 & 83,0 & & 58,9 & 41,1 & \\
\hline \multicolumn{7}{|l|}{ Susceptibilidad } \\
\hline Alta-media & 36,1 & 63,9 & 0,00 & 72,2 & 27,8 & 0,60 \\
\hline Baja-ninguna & 26,7 & 73,3 & & 65,6 & 34,4 & \\
\hline No sabe ${ }^{\star \star}$ & 15,0 & 85,0 & & 66,7 & 33,3 & \\
\hline \multicolumn{7}{|l|}{ Severidad } \\
\hline Alta-media & 27,2 & 72,8 & 0,33 & 66,8 & 33,2 & 0,01 \\
\hline Baja-ninguna & 16,7 & 83,3 & & 33,3 & 66,7 & \\
\hline No sabe ${ }^{\star \star}$ & 13,6 & 86,4 & & 90,9 & 9,1 & \\
\hline \multicolumn{7}{|l|}{ Beneficios } \\
\hline Sí & 26,1 & 73,9 & 0,68 & 69,6 & 30,4 & 0,00 \\
\hline No & 14,3 & 85,7 & & 14,3 & 85,7 & \\
\hline \multicolumn{7}{|l|}{ Barreras* } \\
\hline Sí & 21,0 & 79,0 & 0,00 & 65,2 & 74,8 & 0,02 \\
\hline No & 47,0 & 53,0 & & 81,7 & 18,3 & \\
\hline
\end{tabular}

* 259 datos.

** Esta categoría corresponde a hombres que dijeron no saber su grado de severidad o susceptibilidad respectivamente.

SGSSS = Sistema General de Seguridad Social. 
a los reportados en estudios en Norte América en los que también se usó el MCS 13,18,19,20.

Por el lugar actual del cáncer en la próstata en el perfil epidemiológico de Cali, la información reportada por el estudio es relevante, considerando que identifica aspectos que podrían haber afectado la práctica del EDR en el pasado: el bajo nivel de escolaridad, el estrato socioeconómico, la falta de afiliación al SGSSS, la percepción de susceptibilidad y de barreras. Mientras la intención de práctica puede ser afectada por la falta de afiliación al SGSSS, la percepción de severidad y de barreras y la no percepción de beneficios.

Las diferencias significativas halladas para el antecedente de práctica con respecto al estrato socioeconómico pueden estar relacionadas con el hecho de que los hombres de estrato socioeconómico medio y alto tengan, independiente del estado de afiliación al seguro de salud, mayores posibilidades de acceder a instituciones de salud adicionales que deben garantizar la práctica del EDR en el SGSSS.

Con el fin de eliminar las barreras de acceso a este servicio preventivo en el SGGSS, se hace necesario el desarrollo de acciones dirigidas a garantizar una mayor calidad en la inducción de la demanda del EDR, como el suministro de una información más adecuada y pertinente a un mayor número de hombres, tal como fue requerido por los participantes en el componente cualitativo del estudio 17. Estas acciones deberían enfocarse principalmente hacia población de baja escolaridad y menores ingresos, quienes comparativamente tienen un bajo antecedente e intención de práctica, frente a individuos de mayor nivel de escolaridad y estrato socioeconómico.

Considerando que solamente la mitad de los participantes dijeron haber recibido información sobre el cáncer en la próstata y/o el EDR y dado que en estudios previos se ha encontrado la importancia de la información dada por el médico para inducir la práctica de la tamización en general, y específicamente para el cáncer en la próstata, en futuros estudios se deberían explorar con mayor detalle los factores que desde los proveedores de servicios de salud están determinando la inducción de la demanda del EDR.

Adicionalmente, para mejorar los servicios para la prevención y detección temprana del cáncer es necesario considerar medidas como las planteadas por Dietrich et al. 21, quienes consideran se requiere no sólo de procesos de educación a los médicos, sino aumentar la participación de todo el equipo de salud y contar con adecuadas condiciones logísticas para la pres- tación de este tipo de servicio. Lo anterior se corresponde con el componente cualitativo del estudio, donde se destacan como hallazgos la percepción de que los servicios de salud no están diseñados para la atención de las necesidades de salud de los hombres 17 .

Las diferencias significativas que fueron halladas con respecto a diferentes grados de percepción de susceptibilidad y el antecedente de práctica del EDR, pueden estar vinculadas con la relación que se ha establecido entre el reconocimiento de síntomas y la valoración de susceptibilidad a una enfermedad 22 . Siendo el cáncer en la próstata una patología asintomática en su curso inicial 23 , los individuos tienden a considerarse no susceptibles, lo cual puede llevar a tener una baja disposición de participar en una prueba de tamización. Esta baja disposición puede estar reforzada por la percepción reportada en el componente cualitativo, en la cual los hombres manifiestan que el personal de salud sólo se centra en la atención de síntomas 17.

Otra posible explicación está referida al hecho de que ante eventos como los que afectan la próstata, la percepción de susceptibilidad puede ocupar un segundo plano frente a otros aspectos de la vida cotidiana de los individuos, ante los cuales se creen más susceptibles. Según Berger \& Luckman 24, la realidad cotidiana está mediada por temporalidad y espacio, por lo cual prácticas preventivas como el EDR pueden no ser prioritarias, pues deben competir con acciones cuyos efectos podrían ser percibidos por los individuos como más tangibles e inmediatos.

Las barreras aquí reportadas para la práctica del EDR pueden estar relacionadas con dos aspectos: características socioculturales de los participantes y la forma en la cual se estructuran y prestan los servicios preventivos en salud para los hombres en Colombia. Frente a lo primero, Doyal plantea que producto del proceso de construcción social se ha configurado un prototipo de hombre en el que predominan la percepción de invulnerabilidad y fortaleza; que asume ciertos roles y riesgos, reprime o amputa otros 25. De igual forma, la renuencia del hombre a admitir debilidad o decadencia, o sentir que se reduce su capacidad producto de una enfermedad, puede llevar a que no se busque oportunamente atención en salud, configurando un fenómeno que ha sido denominado "masculinidad marginalizada" 26.

Según Banks 27, los hombres tienen múltiples características que explican su resistencia a buscar ayuda: consideran que su fortaleza se afirma si se mantienen en silencio, los aspec- 
tos relacionados con la salud y enfermedad son considerados de dominio femenino; ellos y la sociedad consideran que sus necesidades de salud son menores. Esto ha derivado en un limitado desarrollo de opciones para la obtención de información y prestación de servicios en salud, al no ser considerados como grupo prioritario de atención, pero también, porque en la planeación de los servicios se ha priorizado la atención a la salud materno-infantil y a algunas patologías de la mujer 28 .

Las limitaciones de este estudio están referidas a su carácter no probabilístico, el cual impide hacer inferencias o establecer causalidad. Una limitación adicional, tiene que ver con el hecho de que la información acerca de la práctica durante la vida del EDR fue autoreportada, lo cual puede sobreestimar el resultado hallado, ante la tendencia de los individuos a dar respuestas con las que esperan satisfacer al encuestador 29. Con esto, el antecedente de práctica EDR puede ser aún más bajo que el descrito.

A pesar de la anterior limitación, de las dos variables dependientes, el antecedente de práctica resulta más útil para efectos de orientar ac- ciones que incrementen las coberturas de práctica del EDR, dado que aunque la intención es una variable proximal del comportamiento futuro, puede ser afectada por múltiples factores que disminuyen su predicción, por lo cual estudios posteriores donde se sigan cohortes de individuos deberán ayudar a establecer la concordancia entre la intención y práctica del EDR.

De otro lado, las mayores fortalezas de este componente cuantitativo fueron que previo a su desarrollo se condujo el componente cualitativo que sirvió para orientar la elaboración del cuestionario, que fue elaborado y aplicado siguiendo un riguroso proceso.

Futuros estudios, de tipo cualitativo y probabilísticos a gran escala, deberán profundizar en las hipótesis plantadas en este estudio exploratorio. Sin embargo, dado el bajo antecedente e intención de práctica del EDR, los resultados pueden contribuir a estructurar acciones que mejoren la oportunidad de acceso a la práctica del EDR, incrementar y mejorar la información que sobre el cáncer en la próstata y práctica del EDR tienen los hombres de Santiago de Cali.

\section{Resumen}

Usando el Modelo de Creencias en Salud se exploraron creencias sobre el examen digital rectal como tamiz para cáncer en la próstata, antecedente e intención de práctica en hombres de 45 a 64 años de edad de Cali, Colombia. A los 267 participantes se les aplicó un cuestionario, previa selección mediante muestreo proporcional por cuotas. Después de un análisis univariado y bivariado se estableció que sólo un 25,8\% de los participantes se había sometido previamente al examen digital rectal y 68,1\% tenía intención de hacerlo en los siguientes 12 meses. Diferencias significativas $(p<0,05)$ fueron halladas entre antecedente de práctica y nivel de escolaridad, estrato socioeconómico, afiliación al sistema de salud, percepción de susceptibilidad a la enfermedad, percepción de barreras y beneficios. Se encontraron diferencias significativas ( $p<0,05)$ entre intención de práctica y afiliación al sistema de salud, percepción de severidad de la enfermedad y percepción de barreras. Dado el bajo antecedente e intención de práctica del examen, los resultados podrían contribuir a mejorar las estrategias para incrementar su práctica a nivel local.

\section{Colaboradores}

D. I. Lucumí-Cuesta fue responsable de escribir cada una de las partes del manuscrito y analizar la base de datos. G. A. Cabrera-Arana contribuyó en la escritura de cada una de las partes del manuscrito.

\section{Agradecimientos}

Al Programa de Subvenciones para Tesis de Postgrado en Salud Pública de América Latina y el Caribe de la Organización Panamericana de la Salud (OPS), que financió este estudio. 


\section{Referências}

1. López J. Mortalidad por cáncer de próstata en el Valle del Cauca, 1993-1998. Boletín Epidemiológico del Valle 1999; 1:29-36.

2. Elhilali M. PSA screening: the bottom line. CMAJ 2000; 162:791-2.

3. Newschaffer C, Otani K, Kathlen M, Penberthy L. Causes of death in elderly prostate cancer and in a comparison nonprostate cancer cohort. J Natl Cancer Inst 2000; 92:613-21.

4. Oliver S, Gunell D, Donovan J. Comparison of trends in prostate-cancer in England and Wales and the USA. Lancet 2000; 355:1788-9.

5. Sadovsky R. Treatment for nonmetastatic prostate cancer. Am Fam Physician 2000; 61. http//proquest.umi.com/pqdweb (accesado el 31/Ene/ 2003).

6. Instituto Nacional de Cancerología de Colombia. Registro institucional de cáncer 1999. Bogotá: Instituto Nacional de Cancerología; 1999.

7. Registro Poblacional de Cáncer de Cali/Departamento de Patología, Facultad de Salud, Universidad del Valle. Cifras de cáncer en Cali 1962-1996. Cali: Registro Poblacional de Cáncer de Cali; 2001.

8. Lu-Yao G, Albertsen P, Stanford J, Stukel T, Walker-Corkery E. Natural experiment examining impact of aggressive screening and treatment on prostate cancer mortality in two fixed cohorts from Seattle area and Connecticut. BMJ 2002; 325:1-6.

9. Gambert S. Prostate cancer: when to offer screening in the primary care setting. Geriatrics 2001; 56:22-31.

10. Ministerio de Salud de Colombia. Resolución 3997 de 1996. http//www.minsalud.gov.co/Archi vos/R3997_96.doc (accesado el 21/Abr/2001).

11. Salud de Colombia. Resolución 412 de 2000. http// http://www. saludcolombia.com/actual/htmlnormas/Res412_00.htm (accesado el 21/Abr/2001).

12. Strecher V, Rosentock I. The health belief model. In: Glanz K, Lewis F, Rimer B, editors. Health behavior and health education: theory, research and practice. 2nd Ed. San Francisco: Jossey Bass; 1997. p. 41-57.

13. Eisen S, Waterman B, Skinner C, Scherrer J, Romeis J, Bucholz K, et al. Sociodemographic and health status characteristics associate with prostate cancer screening in a national cohort of middleaged male veterans. Urology 1999; 53:516-22.

14. Cabrera G, Tascón J, Lucumí D. Creencias en salud: historia, constructos y aportes del modelo. Rev Fac Nac Salud Pública 2001; 19:91-101.

15. Glanz K, Lewis F, Rimer B. Linking theory, research and practice. In: Glanz K, Lewis F, Rimer B, editors. Health behavior and health education: theory, research and practice. 2nd Ed. San Francisco: Jossey Bass; 1997. p. 19-36.
16. Departamento Administrativo Nacional de Estadística. Proyecciones municipales de población. http://www.dane.gov.co/inf_est/series_proyecciones.htm (accesado el 16/Ene/2004).

17. Lucumí D, Cabrera G. Creencias sobre examen digital rectal en hombres de Cali-Colombia: hallazgos cualitativos. Colomb Med 2003; 34:112-9.

18. Carter F, Graham E, Pal N, González E, Roetzhem R. Prostate cancer screening in primary care. South Med J 1999; 92:300-3.

19. Myers R, Wolf T, McKee L, McGrory G, Burgh D, Nelson G, et al. Factors associated with intention to undergo annual prostate cancer screening among African American men in Philadelphia. Cancer 1996; 78:471-9.

20. Edwards Q, Johnson CG, Mason S, Boyle G. Differentiation of the health behavior patterns related to prostate cancer screening among AfricanAmerican men in military settings. Mil Med 2002; 167:374-8.

21. Dietrich A, O’Connor G, Keller A, Carney P, Levy $D$, Whaley F. Cancer: improving early detection and prevention. A community practice randomised trial. BMJ 1992; 304:687-91.

22. Rimer B. Perspectives on intrapersonal theories of health behavior. In: Glanz K, Lewis F, Rimer B, editors. Health behavior and health education: theory, research and practice. 2nd Ed. San Francisco: Jossey Bass; 1997. p. 139-47.

23. Tanagho E, McAninch J. Urología general de Smith. México DF: Manual Moderno; 1997.

24. Berger L, Luckman T. La construcción social de la realidad. Buenos Aires: Amorrou Editores; 1991.

25. Doyal L. Sex, gender and health: the need for a new approach. BMJ 2001; 323:1061-3.

26. Sabo D. Comprender la salud de los hombres: un enfoque relacional y sensible al género. Washington DC: Organización Panamericana de la Salud/ Harvard Center of Population and Development Studies; 2000.

27. Banks I. No man's land: men, illness, and the NHS. BMJ 2001; 323:1058-60.

28. Kiss A, Meryn S. Effect of sex and gender on psychosocial aspects of prostate and breast cancer. BMJ 2001; 323:1055-8.

29. Newell S, Girgis A, Sanson-Fisher R, Savolainen $\mathrm{N}$. The accuracy of self-reported health behaviors and risk factors relating to cancer and cardiovascular disease in the general population. A critical review. Am J Prev Med 1999; 17:211-29.

Recibido el 13/Sep/2004

Versión final presentada el 30/Mar/2005

Aprobado el 08/Abr/2005 